\title{
Short Communication: Trends in Transmitted Drug Resistance in Treatment-Naive HIV Patients in Korea
}

\author{
Eun Ju Jung, ${ }^{1}$ Sun Hee Lee, ${ }^{2}$ Shinwon Lee, ${ }^{2}$ Jeong Eun Lee, ${ }^{2}$ Soon Ok Lee, ${ }^{2}$ \\ Sohee Park, ${ }^{2}$ Hyung-Hoi Kim, ${ }^{3}$ Jin Suk Kang, ${ }^{4}$ and Heung jung Woo ${ }^{1}$
}

\begin{abstract}
Transmitted drug resistance (TDR) in treatment-naive HIV patients can contribute to failure of initial antiretroviral therapy. In Korea, there has been a gradual increase in TDR, but the recent increase in the use of integrase strand transfer inhibitors (INSTIs) could affect TDR trends and INSTI resistance mutations. We evaluated the patterns of TDR in newly diagnosed HIV patients over time from 2011 to 2019. We analyzed the genotypic resistance of strains in 336 patients and sequenced the integrase gene in 71 among 336 subjects. The overall prevalence of TDR was 5.9\% (20 of 336 patients), and it showed a tendency to increase over time (5.1\% in 2011-2013, 6.1\% in 2014-2016, and 7.2\% in 2017-2019; $p=.3018)$. Furthermore, non-nucleoside reverse transcriptase inhibitor resistance showed a marginally significant increase over time $(1.45 \%$ in $2011-2013$, $3.48 \%$ in $2014-2016$, and $6.02 \%$ in $2017-2019$; $p=.0505$ ). Regarding transmitted INSTI resistance mutation, there were no major INSTI resistance mutations but several accessory INSTI resistance mutations and predominant natural polymorphisms. This study shows several significant changes in TDR and suggests the importance of continuous surveillance of TDR and genetic variation in the integrase region.
\end{abstract}

Keywords: HIV, transmitted resistance, resistance mutation, integrase strand transfer inhibitor, polymorphisms

$\mathbf{T}$ HE INTRODUCTION OF highly active antiretroviral therapy (HAART) has significantly reduced HIV-related morbidity and mortality. However, emergence of drugresistant strains limits the efficiency of HAART and leads to treatment failure. These drug resistance mutations can be transmitted to treatment-naive patients and adversely affect virologic response to initial antiretroviral therapy. ${ }^{1} \mathrm{Re}-$ cently, guidelines emphasize that HAART be started immediately or as soon as possible after diagnosis to reduce the risk of HIV transmission and to improve the rate of virologic suppression among persons with HIV. Also, a twodrug regimen of dolutegravir plus lamivudine has been newly added to the list of initial regimens for people with HIV, and a two-drug long-acting injectable regimen is being developed. These recent circumstances suggest that surveillance of transmitted drug resistance (TDR) is important to provide appropriate guidelines for antiretroviral therapy.
In Korea, the prevalence of TDR is lower than in the United States and Europe. ${ }^{2-5}$ However, use of antiretroviral drugs is increasing with the recent increase in number of Korean HIV patients, and integrase strand transfer inhibitors (INSTIs) are increasingly being used after introduction of raltegravir (Isentress) in 2010 and release of the tablet Stribild including elvitegravir in 2014. Next, Triumeq, Genvoya, and Biktarvy, the tablets including INSTIs were sequentially introduced into Korea in 2015, 2017, and 2019, respectively. Wide use of INSTIs is expected to change the overall TDR in treatment-naive patients. Emergence of an INSTI resistance mutation is being reported, ${ }^{6}$ as is resistance to dolutegravir in treatment-naive patients. ${ }^{7}$ Most recently, there was a study that first reported a major INSTI resistance mutation, E92Q, in treatment-naive patients in Korea. ${ }^{8}$ These results suggest the importance of continuous monitoring of TDR including INSTI resistance mutations.

\footnotetext{
${ }^{1}$ Division of Infectious Diseases, Department of Internal Medicine, Hallym University College of Medicine, Chuncheon, Republic of Korea.

${ }^{2}$ Division of Infectious Diseases, Department of Internal Medicine, Pusan National University School of Medicine and Medical Research Institute, Pusan National University Hospital, Busan, Republic of Korea.

${ }^{3}$ Department of Laboratory Medicine, BioMedical Informatics Unit, Pusan National University School of Medicine, Busan, Republic of Korea.

${ }^{4}$ Division of Infectious Diseases, Department of Internal Medicine, Inje University College of Medicine, Busan, Republic of Korea.
} 
Our subjects were treatment-naive HIV patients visiting Pusan National University Hospital from February 2011 to December 2019. The hospital is a 1,351-bed universityaffiliated teaching hospital and provides HIV care for affected patients in the southeastern region of Korea. A total of 336 patients were enrolled, and their medical records were reviewed for epidemiological and clinical data. The study protocol and standardized case record forms were approved by our institutional review board. HIV-1 genotyping was performed using the ViroSeq HIV-1 Genotyping System (Abbott Laboratories, USA). HIV-1 RNA was extracted from plasma following the manufacturer's protocol, and the entire protease and reverse transcriptase genes of the pol region were amplified by reverse transcription polymerase chain reaction. We sequenced the integrase gene in 71 among 336 subjects. We used the ViroSeq ${ }^{\mathrm{TM}}$ HIV-1 integrase genotyping kit (Abbott Laboratories) for 19 subjects from 2011 to 2016, whereas sequencing of 52 subjects was outsourced to Seoul Clinical Laboratories starting in 2017. Antiretroviral resistance mutations were identified by the Stanford HIV Drug Resistance Database (version 8.9).

Demographic and clinical characteristics of 336 patients are summarized in Table 1. Comparing the characteristics between the patients grouped by diagnosed years, the mean age of the more recently diagnosed patients was significantly lower (49 years in 2011-2013, 45 years in 2014-2016, and 42 years in 2017-2019; $p=.002$ ). The patients who were heterosexual decreased from $44.9 \%$ (2011-2013) to $33.7 \%$ (2017-2019), but men who had sex with men increased over time during the study period (48.6\% in $2011-2013,63.9 \%$ in 2017-2019) ( $p=.017)$. Among 71 patients of whom the integrase gene was sequenced, the majority belonged in the group of more recently diagnosed patients with HIV. Two patients $(2.8 \%)$ were diagnosed in 2011-2013, 17 (23.9\%) in 2014-2016, and 52 (73.2\%) in 2017-2019. Other baseline characteristics (gender, age, CD4 cell count, and viral load) were not significantly different among 71 patients. Genotypic resistance analysis showed 22 surveillance of transmitted drug resistance mutations (SDRMs) in 20 subjects. The overall prevalence of TDR was 5.9\% (20 of 336 patients), and SDRM associated with individual drug classes was $2.1 \%$ (7 of 336) in nucleoside reverse transcriptase inhibitor (NRTI), 3.3\% (11 of 336) in non-nucleoside reverse transcriptase inhibitor (NNRTI), and 1.2\% (4 of 336) in protease inhibitor (PI). There were no major INSTI resistance mutations $(\mathrm{T} 66 \mathrm{~A} / \mathrm{I} / \mathrm{K}$, E92Q, G118R, E138K/A/T, G140S/A/C, Y143R/C/H, S147G, $\mathrm{Q} 148 \mathrm{H} / \mathrm{R} / \mathrm{K}, \mathrm{N} 155 \mathrm{H}$, and R263K). There was one multidrugresistant strain harboring SDRM in both NRTI and NNRTI (D67N and K219E in NRTI and G190A in NNRTI).

To evaluate the trend in the prevalence of TDR over time, we conducted a Cochran-Armitage trend test. Although the $p$ value was not statistically significant, the overall prevalence of TDR showed a tendency to increase during the study period $(5.1 \%$ in $2011-2013,6.1 \%$ in $2014-2016$, and $7.2 \%$ in 2017-2019; $p=.3018$ ) (Fig. 1A). We found that NNRTI resistance marginally significantly increased over time $(1.45 \%$ in $2011-2013,3.48 \%$ in $2014-2016$, and $6.02 \%$ in $2017-$ $2019 ; p=.0505$ ) (Fig. 1A). Furthermore, we subanalyzed the prevalence of TDR according to age, gender, transmission route, time interval between diagnosis and study, subtype (B or non-B), and CD4 T lymphocyte (CD4) cell count. As age increased, the overall prevalence of TDR tended to decrease and NNRTI resistance decreased significantly $(p=.0151)$ (Fig. 1B). In contrast, other factors did not show a significant association with TDR except CD4 cell count. As CD4 cell count increased, NRTI resistance decreased and PI resistance increased significantly ( $p=.0335$ for NRTI resistance; $p=.0437$ for PI resistance).

With regard to TDR for INSTIs, there were no major mutations, although accessory mutations E157Q and L74I

Table 1. Characteristics of Treatment-Naive HIV-Infected Patients from 2011 to 2019 (N=336)

\begin{tabular}{|c|c|c|c|c|c|}
\hline \multirow[b]{2}{*}{ Characteristics } & \multicolumn{3}{|c|}{ Year } & \multirow[b]{2}{*}{ Total $(\mathrm{n}=336)$} & \multirow[b]{2}{*}{$\mathrm{p}^{\mathrm{a}}$} \\
\hline & $2011-2013(\mathrm{n}=138)$ & $2014-2016(\mathrm{n}=115)$ & $2017-2019(\mathrm{n}=83)$ & & \\
\hline $\begin{array}{l}\text { Age (years), } \\
\text { mean (range) }\end{array}$ & $49(23-84)$ & $45(24-82)$ & $42(22-79)$ & $46(22-84)$ & .002 \\
\hline Male $(\%)$ & $124(89.9)$ & $102(88.7)$ & $81(97.6)$ & 307 (91.4) & .065 \\
\hline $\begin{array}{l}\text { Risk factor for } \\
\text { transmission }(\%)\end{array}$ & & & & & $.017^{\mathrm{b}}$ \\
\hline Heterosexual & $62(44.9)$ & $48(41.7)$ & $28(33.7)$ & $138(41.1)$ & \\
\hline MSM & 67 (48.6) & $54(47.0)$ & $53(63.9)$ & $174(51.8)$ & \\
\hline IV drug & $2(1.4)$ & $1(0.9)$ & 0 & $3(0.9)$ & \\
\hline MSM with IV drug & $4(2.9)$ & $1(0.9)$ & 0 & $5(1.5)$ & \\
\hline Unidentified & $3(2.2)$ & $11(9.6)$ & $2(2.4)$ & $16(4.8)$ & \\
\hline $\begin{array}{l}\text { Plasma viral load } \\
\text { (log copies/mL), } \\
\text { mean } \pm \text { SD (range) }\end{array}$ & $4.9 \pm 0.9(1.3-7.0)$ & $4.9 \pm 0.8(3.3-7.0)$ & $5.0 \pm 0.9(3.0-8.0)$ & $4.9 \pm 0.9(1.3-8.0)$ & .813 \\
\hline $\begin{array}{l}\text { CD } 4 \text { cell count } \\
\left(\text { cells } / \mathrm{mm}^{3}\right)\end{array}$ & $279 \pm 193(1-807)$ & $321 \pm 284(5-1625)$ & $266 \pm 211(1-848)$ & $290 \pm 232(1-1625)$ & .193 \\
\hline HIV-1 subtype (\%) & & & & & .132 \\
\hline Subtype B & $116(84.1)$ & $85(73.9)$ & $64(77.1)$ & $265(78.9)$ & \\
\hline Non-B & $22(15.9)$ & $30(26.1)$ & 19 (22.9) & $71(21.1)$ & \\
\hline
\end{tabular}

${ }^{\mathrm{a} O n e-w a y ~ A N O V A}$ for continuous variable, chi-squared test for categorical variable.

'Fisher's exact test.

ANOVA, analysis of variance; IV, intravenous; MSM, men who have sex with men; SD, standard deviation. 


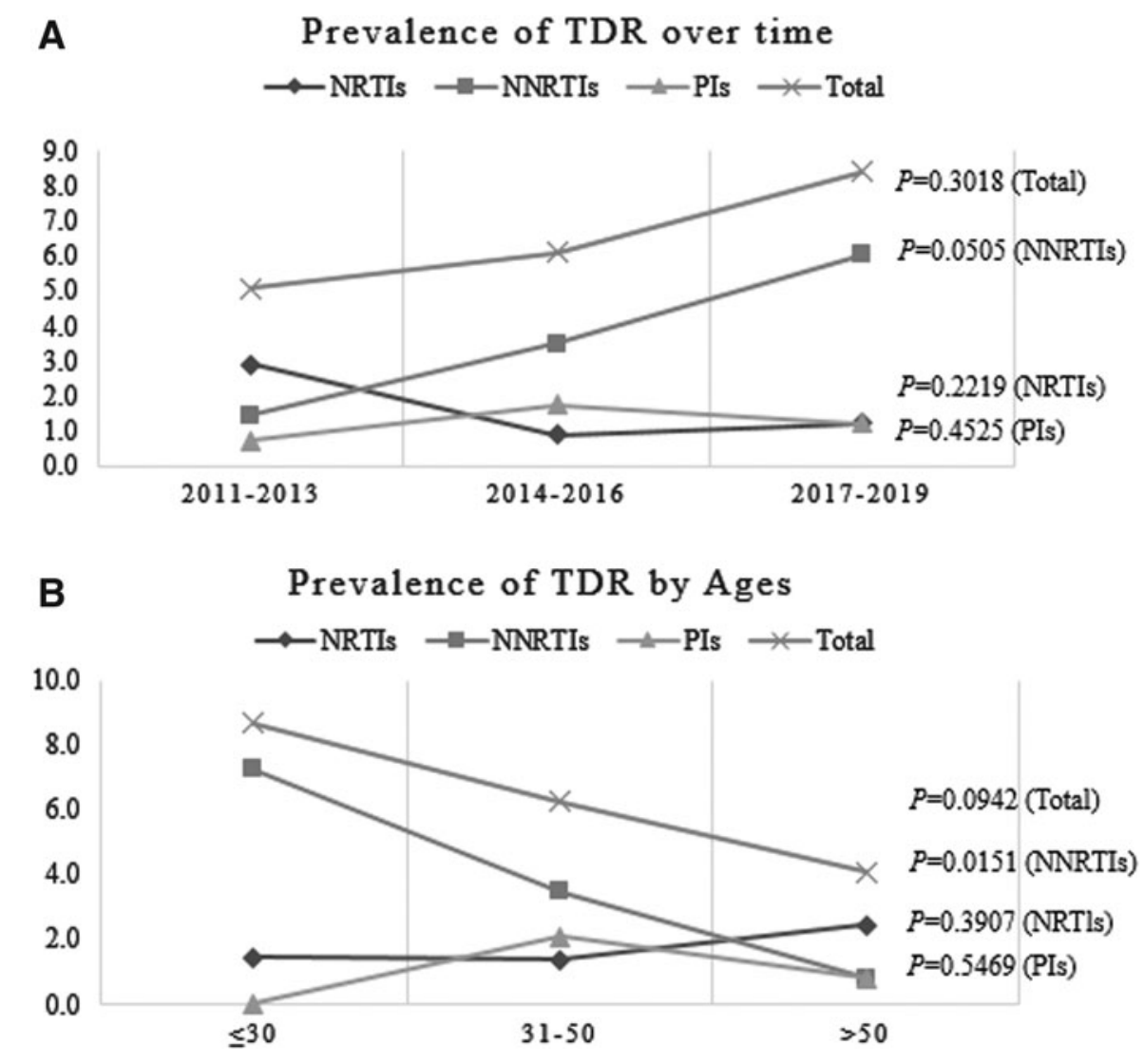

FIG. 1. Trend of the prevalence of TDR. (A) We found that the overall prevalence of TDR and NNRTI resistance showed increasing tendency over time using Cochran-Armitage trend test. (B) In contrast, the overall prevalence of TDR and NNRTI resistance showed decreasing tendency as age increases. NNRTI, nonnucleoside reverse transcriptase inhibitor; NRTI, nucleoside reverse transcriptase inhibitor; TDR, transmitted drug resistance.

were found in $11.6 \%$ (5 of 71) and 9.3\% (4 of 71) of patients, respectively. Furthermore, we identified genetic variation in the integrase gene in 71 strains and assessed which substitutions showed a Stanford HIV database mutation score $\geq 10$ in at least one INSTI. Except E157Q, no substitutions with an HIV database mutation score $\geq 10$ were detected in the 71 subjects.

From our results, although it was not statistically significant, the trend for TDR increased over time and this trend was similar to the results of previous studies in Korea. Reviewing the studies, the prevalence of TDR was $\sim 5 \%$ during 1999 $2007,,^{3,5,9}$ gradually increased to $6-7 \%$ during 2009-2012, ${ }^{10}$ and reached $8.4 \%$ during $2013-2014 .{ }^{11}$ These findings suggest an increasing prevalence for TDR among newly diagnosed HIV patients and indicate that continuous monitoring of TDR is important in Korea.

Also, we found that NNRTI resistance increased significantly during the study period. The increasing trend in transmitted NNRTI resistance is in agreement with recent data from Europe. ${ }^{12,13}$ These changes can be explained by prescription practices and properties of mutations. NNRTI has been popularly used as first-line treatment in the past. However, these drugs have a low genetic barrier to drug resistance and can select resistant viruses through failed treatments. M184V has a strong effect on replication capacity and, if transmitted, reverts back to wild-type rapidly. ${ }^{14}$ In contrast, the $\mathrm{K} 103 \mathrm{~N}$ mutation has a limited effect on viral replication capacity that persists for long periods after transmission. ${ }^{13}$ The K103N was the most common drug resistance mutation in our study ( 8 of 336 patients, $2.4 \%$ ), and this result coincides with another study performed in 2015 in Korea. ${ }^{11}$ That study revealed the K103N mutation in $6.1 \%$
(8 of 131 patients) of patients and was the most commonly detected TDR mutation. The authors suggested that the increase in K103N TDR was likely related to increased use of NNRTI, especially efavirenz. ${ }^{11}$

Interestingly, the prevalence of TDR and NNRTI resistance tended to decrease as age increased (Fig. 1B). Taking this with the mentioned result (Fig. 1A), we can suggest that patient age at diagnosis became younger over the years in this study. We found that the mean age of the more recently diagnosed patients was lower, as given in Table 1 . The proportion of patients $<30$ years was $8.7 \%$ in $2011-2013$ but increased to $33.7 \%$ in 2017-2019. Conversely, patients $>50$ years represented $44.2 \%$ of patients in 2011-2013 but decreased to $25.3 \%$ in $2017-2019(p<.0001$, data are not shown). Reviewing the annual report on HIV from Center for Disease Prevention in Korea, the age group of 20-39 years accounted for the majority $(60 \%)$ of newly diagnosed patients and this trend increased over time. Taken together, these results indicate that the proportion of younger persons is increasing among newly diagnosed HIV patients, as is the overall prevalence of TDR and NNRTI resistance.

Unexpectedly, some significant changes were observed in NRTI and PI resistance with CD4 count distribution. There is a limitation resulting from our small sample size, but this result seems to show a significant $p$ value because of a disparity in CD4 count distribution between age groups. In our study, there was no significant disparity in CD4 count distribution by years, but we found a tendency of lower CD4 count in older patients ( $p<.0001$, data are not shown). Older people appear to have lower CD4 count at seroconversion and steeper CD4 count decline over time. ${ }^{15}$ 
There was no major INSTI resistance among the 71 patients, but we identified integrase gene polymorphisms. Lone natural polymorphisms have little or no effect on INSTI susceptibility. However, there have been several relevant studies that considered their potential contribution to susceptibility. ${ }^{16-19}$ One previous study explained natural polymorphisms in Korean strains. ${ }^{9}$ Similar to that study, ${ }^{9}$ we observed polymorphic sites V31I, T124N, T125A, and V201I at high frequency ( $>50 \%$ in 71 subjects) in our study. Although the substitutions showed a slight difference in prevalence, predominant polymorphisms were observed at a similar frequency, and we identified no new unique substitutions compared with reports from other countries. ${ }^{16,18,20}$ Among our highly variable substitutions (E11D, L28I, V31I, S39C, L101I, I113V, T124N, T125A, I135V, G163E, V201I, and D256E), none is known to contribute significant resistance to INSTIs. Nevertheless, V201I has been reported as a natural polymorphism associated with INSTI resistance (S-1360) and a typical signature for subtypes C and F. ${ }^{16,17}$ Thus, the impact of natural polymorphisms on the efficiency of antiviral therapy remains unclear. However, previous studies suggest that natural polymorphisms partially compensate for resistance mutations, resulting in reduced susceptibility to antiretroviral therapy.

In our patients with TDR $(n=20)$, the antiretroviral therapy was started in 16 patients except for 4 ( 2 patients were transferred to another medical center, 1 patient died of pneumonia before initiation of therapy, and 1 patient refused therapy). Predominantly, INSTI plus two NRTI regimens was used as first-line therapy in 12 patients $(75 \%)$. Among seven patients with NNRTI resistance, all were prescribed INSTI-based regimens as first therapy. Among five patients with NRTI resistance, four patients received INSTI-based or PI-based regimens and the other with active tuberculosis received NNRTI-based regimen. Among four patients with PI resistance, three patients received INSTI-based regimen and one patient received lopinavir/ritonavir with two NRTIs. Among 16 patients, $93.7 \%$ (15 of 16) reached the viral load limit $(<50$ copies/mL) within 6 months after start of therapy, and the other reached the viral load limit between 6 and 12 months after start of therapy.

This study was limited by its single-center setting and small sample size. However, our research institution is a national university-affiliated hospital located in the second largest city in South Korea. This representative position should compensate for the limitation. Another limitation is the various time intervals between diagnosis and study. We could not enroll only patients diagnosed with acute or recent HIV infection. However, the majority of patients $(55 \%)$ had a short time interval between diagnosis and study ( $<1$ month), which again should compensate for the limitation.

In conclusion, the overall prevalence of TDR tended to increase from 2011 to 2019 in a single-center cohort in Busan, Korea. There was a marked increase in resistance to NNRTI, which could affect antiretroviral therapy. For efficient treatment, a baseline resistance test before implementation of treatment is recommended. Also, possible transmitted INSTI resistance mutations and failure of antiretroviral therapy must be considered given the increased use of INSTIs. No major integrase inhibitor resistance was found in this study, but there were several polymorphisms that conferred potential resistance to antiretroviral drugs. These polymorphisms suggest that resistance to some antiretroviral drugs may occur faster in patients harboring these polymorphic variants, particularly under drug selection pressure. Therefore, we suggest surveillance of integrase inhibitor resistance mutations and further study on integrase genetic variants.

\section{Author Disclosure Statement}

No competing financial interests exist.

\section{Funding Information}

No funding was received for this article.

\section{References}

1. Little SJ, Holte S, Routy JP, et al:: Antiretroviral-drug resistance among patients recently infected with HIV. N Engl J Med 2002;347:385-394.

2. won Park S, Kim HB, ju Choi Y, Kim NJ, don Oh M, won Choe K: Genotypic resistance of antiretroviral drugs among drug-naive HIV type 1 patients with the background of long-term access-easy zidovudine therapy. AIDS Res Hum Retroviruses 2003;19:1039-1043.

3. Bang JI, Song KH, Kim S-H, et al.: Prevalence of primary antiretroviral resistance: Trends in Korea. AIDS Res Hum Retroviruses 2008;24:83-85.

4. Baxter JD, Dunn D, White E, et al.: Global HIV-1 transmitted drug resistance in the INSIGHT strategic timing of antiretroviral treatment (START) trial. HIV Med 2015;16 Suppl 1:77-87.

5. Choi J-Y, Kim E-J, Park YK, et al.: National survey for drug-resistant variants in newly diagnosed antiretroviral drug-naive patients with HIV/AIDS in South Korea: 19992005. J Acquir Immune Defic Syndr 2008;49:237-242.

6. Chang SY, Lin P-H, Cheng C-L, et al:: Prevalence of integrase strand transfer inhibitors (INSTI) resistance mutations in Taiwan. Sci Rep 2016;6:35779.

7. Raesima MM, Ogbuabo CM, Thomas V, et al.: Dolutegravir use at conception-Additional surveillance data from Botswana. N Engl J Med 2019;381:885-887.

8. Jeong W, Jung IY, Choi H, et al.: Integrase strand transfer inhibitor resistance mutations in antiretroviral therapy-naive and treatment-experienced HIV patients in South Korea. AIDS Res Hum Retroviruses 2019;35:213-216.

9. Kim JY, Kin EJ, Choi JY: Genetic variation of the HIV-1 integrase region in newly diagnosed anti-retroviral drugnaïve patients with HIV/AIDS in Korea. Clin Microbiology and Infection 2010;17.

10. Park M, Kee MK, Rhee JE, et al.: The trend of transmitted drug resistance in newly diagnosed antiretroviral-naive HIV/AIDS patients during 1999-2012 in South Korea. J Clin Virol 2016;81:53-57.

11. Chin BS, Shin HS, Kim G, et al.: Short communication: Increase of HIV-1 K103N transmitted drug resistance and its association with efavirenz use in South Korea. AIDS research and human retroviruses 2015;31:603-607.

12. Bartmeyer B, Kuecherer C, Houareau C, et al.: Prevalence of transmitted drug resistance and impact of transmitted resistance on treatment success in the German HIV-1 seroconverter cohort. PLoS One 2010;5:e12718.

13. Dineke Frentz DAVdV, Ana B Abecasis, Jan Albert, et al.: Increase in transmitted resistance to non-nucleoside reverse transcriptase inhibitors among newly diagnosed HIV-1 infections in Europe. BMC Infect Dis 2014;14:407. 
14. Jain V, Sucupira MC, Bacchetti $\mathrm{P}$, et al.: Differential persistence of transmitted HIV-1 drug resistance mutation classes. J Infect Dis 2011;203:1174-1181.

15. Lodi S, Phillips A, Touloumi G, et al.: Time from human immunodeficiency virus seroconversion to reaching CD4+ cell count thresholds $<200,<350$, and $<500$ cells $/ \mathrm{mm}^{3}$ : Assessment of need following changes in treatment guidelines. Clin Infect Dis 2011;53:817-825.

16. Lataillade M, Chiarella J, Kozal MJ: Natural polymorphism of the HIV-1 integrase gene and mutations associated with integrase inhibitor resistance. Antivir Ther 2007;12:563570.

17. Passes CB, Guimarães ML, Fernandez Saada Lima C, et al.: Lack of primary mutations associated with integrase inhibitors among HIV-1 subtypes B, C, and F circulating in Brazil. J Acquir Immune Defic Syndr 2009;51.

18. Ceccherini-Silberstein F, Malet I, Fabeni L, et al. Specific HIV-1 integrase polymorphisms change their prevalence in untreated versus antiretroviral-treated HIV-1-infected patients, all naive to integrase inhibitors. J Antimicrob Chemother 2010;65:2305-2318.
19. Rhee SY, Liu TF, Kiuchi M, et al: Natural variation of HIV-1 group M integrase: Implications for a new class of antiretroviral inhibitors. Retrovirology 2008;5:74.

20. Casadella M, van Ham P M, Noguera-Julian M, et al: Primary resistance to integrase strand-transfer inhibitors in Europe. J Antimicrob Chemother 2015;70:2885-2888.

Address correspondence to: Sun Hee Lee, MD, PhD Division of Infectious Diseases Department of Internal Medicine Pusan National University School of Medicine and Medical Research Institute Pusan National University Hospital 179 Gudeok-ro, Seo-gu Busan 49241 Republic of Korea E-mail: zzanmery@gmail.com 